Mariana de Almeida Pinto Borges' Maria LUCIA Elias Pires ${ }^{2}$ Denise Lette Maia Monteiro ${ }^{3}$ SueLY RodRIGUES dOS SANTOS ${ }^{4}$

Relato de caso

Palavras-chave

Útero/anormalidades Vagina/anormalidades

Rim/anormalidades

Aberrações cromossômicas Anormalidades múltiplas/genética Amenorréia

Ductos de Muller/anormalidades Relatos de casos

Keywords

Uterus/abnormalities Vagina/abnormalities Kidney/abnormalities

Chromosome aberrations Abnormalities, multiple/genetics Amenorrhea

Mullerian ducts/abnormalities Case reports

\section{Forma atípica da síndrome de Mayer-Rokitansky- Kuster-Hauser com malformação renal e displasia cervicotorácica (associação de MURCS)}

\author{
Atipical form of Mayer-Rokitansky-Kuster-Hauser syndrome with \\ renal malformation and skeletal abnormalities (MURCS association)
}

\section{Resumo}

A forma atípica e mais severa da síndrome Mayer-Rokitansky-Kuster-Hauser (MRKH) ou MRKH tipo II é também conhecida como associação de MURCS, cujo mnemônico significa aplasia/hipoplasia mülleriana (MU), malformação renal (R) e displasia cervicotorácica (CS). Acomete pacientes do sexo feminino com cariótipo e função ovariana normais, acarretando amenorreia primária. Apresenta incidência de 1:50.000, subestimada pelo diagnóstico tardio e etiologia mal definida. Descrevemos um caso em criança e outro em adolescente, com o objetivo de predizer o diagnóstico ainda na infância, antes da instalação do quadro de amenorreia; as pacientes apresentavam em comum malformação renal, agenesia ou hipoplasia de derivados müllerianos e anomalias vertebrais, configurando o diagnóstico de MURCS. A relevância do estudo é mostrar a necessidade de prosseguir a investigação na presença de algum dos sinais da doença, pesquisando anormalidades correlatas, a fim de se estabelecer o diagnóstico precocemente e, consequentemente, orientar pacientes e seus familiares quanto à melhor forma de condução do caso, incluindo aconselhamento genético.

\section{Abstract}

The atypical and more severe form of Mayer-Rokitansky-Kuster-Hauser syndrome (MRKH) or MRKH type II is also known as MURCS association, an acronym meaning aplasia/hypoplasia of Müllerian ducts (MU), congenital renal dysplasia (R) and cervico-thoracic dysplasia (CS). It affects female patients with normal karyotype and ovarian function, evolving to primary amenorrhea. It has an incidence of 1:50,000, but it is underestimated due to late diagnosis and undefined etiology. We describe the cases of a child and an adolescent in order to predict the diagnosis even in childhood, before the onset of amenorrhea. Patients had in common renal malformation, agenesis or hypoplasia of Müllerian derivatives and vertebral anomalies, establishing the diagnosis of MURCS. The relevance of this paper is to show the importance of further investigation when some of pathologic signs are present, researching correlated abnormalities in order to establish an early diagnosis and consequently to provide guidance to the patients and their families about the best way to conduct the case, including genetic counseling.
Correspondêncio

Mariana de Almeida Pinto Borges Rua Mariz e Barros 775 - Tijuce CEP: 20270-004

Rio de Janeiro (RJ), Brasil

Recebido

$19 / 10 / 2010$

Aceito com modificaçōes
Trabalho realizado no Hospital Universitário Gaffrée e Guinle da Universidade Federal do Estado do Rio de Janeiro - UNIRIO - Rio de Janeiro (RJ), Brasil.

'Programa de Pós-graduação do Serviço de Endocrinologia do Hospital Universitário Gaffrée e Guinle, Universidade Federal do Estado do Rio de Janeiro - UNIRIO - Rio de Janeiro (RI), Brasil.

2 Escola de Medicina e Cirurgia Universidade Federal do Estado do Rio de Janeiro - UNIRIO - Rio de Janeiro (RI), Brasil.

${ }^{3}$ Centro Universitário Serra dos Órgãos - UNIFESO - Teresópolis (RJ), Brasil; Faculdade de Ciências Médicas, Universidade Estadual do Rio de Janeiro - UERJ - Rio de Janeiro (RJ), Brasil.

${ }^{4}$ Curso de Pós-graduação em Endocrinologia do Hospital Universitário Gaffrée e Guinle, Universidade Federal do Estado do Rio de Janeiro - UNIRIO - Rio de Janeiro (RJ), Brasil; Ambulatório de Genética do Hospital Universitário Gaffrée e Guinle, Universidade Federal do Estado do Rio de Janeiro - UNIRIO - Rio de Janeiro (RJ), Brasil. 


\section{Introdução}

A síndrome Mayer-Rokitansky-Kuster-Hauser (MRKH) é caracterizada por aplasia congênita do útero e dois terços superiores da vagina em mulheres com cariótipo e características sexuais secundárias normais, apresentando incidência de 1:4.500 meninas ${ }^{1}$. A MRKH pode ocorrer de forma isolada (tipo I) ou associada à malformação renal, vertebral e, em alguns casos, defeitos cardíacos ou auditivos ${ }^{2,3}$, configurando a MRKH tipo II ou associação de MURCS, com incidência de 1:50.000 meninas ${ }^{4}$. A ausência de dois terços proximais da vagina e ausência ou hipoplasia uterina ocorre em $96 \%$ dos casos, agenesia unilateral ou ectopia de um ou ambos os rins ( $88 \%$ dos casos); hidronefrose e rim em ferradura também podem ser encontrados. Já as anomalias de esqueleto, como defeito vertebral, envolvendo segmento cervical e/ou torácico, especialmente entre C5-T1, ocorrem em $80 \%$ dos casos, assim como assimetria, fusão de vértebras ou vértebra em cunha, escoliose e anomalia de Klippel-Feil (fusão de vértebras cervicais). Há relato na literatura da malformação da junção occipito-atlanto-axial, causando hiperostose anquilosante senil vertebral em adolescente com MURCS, com início precoce de limitações na mobilidade da cabeça associadas à rigidez significativa ao longo da coluna vertebral ${ }^{5}$. Entre os defeitos menos frequentes, estão os auditivos e as anomalias cardíacas, cuja severidade é variável ${ }^{6}$.

A constituição cromossômica é $46, \mathrm{XX}$, embora tenham sido descritos casos de disgenesia gonadal XY ou monossomia $\mathrm{X}$ com os defeitos congênitos anteriormente descritos ${ }^{6}$.

A associação de MURCS/MRKH parece ocorrer de forma esporádica, mas há relatos de casos familiais ${ }^{7}$, com herança autossômica dominante, penetrância incompleta e expressividade variável ${ }^{6,8}$. Apesar da etiologia ainda ser mal definida, as malformações encontradas sugerem defeitos no campo de desenvolvimento, durante a embriogênese dos órgãos afetados, a partir da quarta semana de gestação ${ }^{9-11}$. A patogênese está intimamente associada à regressão dos ductos de Müller (paramesonéfricos), não tendo sido encontradas mutações ou polimorfismos no gene codificador do hormônio antimülleriano (HAM) e em seu receptor, como também não foi demonstrado aumento na concentração sérica e na expressão de $\mathrm{HAM}^{3,7}$.

O diagnóstico é tardio na maioria dos casos, sendo a amenorreia primária o principal motivo pela procura do atendimento, o que posterga a detecção da patologia para a época em que, geralmente, ocorre a menarca ou mesmo para a fase adulta. Apesar disso, existem casos descritos na literatura com diagnóstico intraútero, em recém-nascida e aos 7 meses de vida ${ }^{2,12-14}$.

O objetivo do estudo foi relatar dois casos de associação de MURCS, ressaltando a possibilidade de se predizer o diagnóstico ainda na infância, antes da instalação do quadro de amenorreia, principalmente quando outras malformações estiverem associadas, e chamar a atenção dos profissionais envolvidos no atendimento a essas pacientes, que, diante da presença de um dos sinais da doença, impõe-se a realização de procedimentos que identifiquem anormalidades correlatas, a fim de se estabelecer o diagnóstico precocemente, possibilitando traçar a melhor forma de condução do caso.

\section{Métodos}

Foi realizada ampla revisão da literatura médica disponível por meio de pesquisa na mídia digital, utilizando-se as bases de dados: MEDLINE (por meio do PubMed), Google acadêmico, SciELO e LILACS. Para identificação de publicações no MEDLINE, foi utilizada a seguinte estratégia de busca: (murcs [All Fields] AND (“association”[MeSH Terms] OR “association”[All Fields])) AND ("humans"[MeSH Terms] AND ("women" [MeSH Terms] OR "female" [MeSH Terms])), sendo encontrados 45 artigos. Para a busca em outras bases, foram utilizados os termos "MURCS association" e "associação de MURCS". A busca resultou no total de 54 artigos, publicados entre 1979 e 2011, que foram avaliados por dois revisores, sendo selecionados 17 artigos além de três livros-textos para a confecção desse estudo, visando à discussão dos casos relatados.

Todas as informações descritas no relato dos casos foram obtidas após consentimento informado e assinado pelas mães das pacientes, seguindo as orientações do Código de Ética Médica do Conselho Federal de Medicina do Brasil (2009) e observando os princípios éticos para pesquisa médica envolvendo seres humanos, compilados na Declaração de Helsinki e corrigida pela $52^{\mathrm{a}}$ Assembleia Geral da Associação Médica Mundial em Edimburgo (2009).

\section{Relato dos casos}

\section{Primeiro caso}

Paciente é filha de pais saudáveis, jovens e não consanguíneos, nascida de parto vaginal, a termo, com peso e estatura compatíveis com a idade gestacional, foi encaminhada ao nosso serviço aos 6 anos de idade, para investigar anomalia de Klippel-Feil. Ultrassonografia (USG) realizada com 22 semanas de gestação revelou imagem cística anexial à esquerda, que, no curso da 
investigação, aos 6 anos, demonstrou tratar-se de rim único ectópico, localizado em fossa ilíaca esquerda, vincariante, mas com função normal e topografia comprovada em USG posterior (Figura 1C). Os dois achados fundamentaram a solicitação da USG (Figura 1B) e tomografia computadorizada (TC) pélvicas, que não evidenciaram estruturas müllerianas (útero e trompas). Ao exame físico, apresentava desenvolvimento intelectual normal, epicanto, telecanto, hipertelorismo ocular, nariz pequeno, aletas hipoplásicas, palato alto e estreito, pescoço curto e alado, gradil costal a esquerda saliente, tórax largo e curto, polegares largos, unhas em vidro de relógio, clinodactilia no quartoquirodáctilo esquerdo e mancha "café com leite" em região axilar posterior direita. Atualmente, aos 11 anos, desenvolvimento puberal M3P2G2 pelos critérios de Tanner ${ }^{15}$, estatura de $1,40 \mathrm{~m}(\mathrm{P} 25)^{16}$ e peso de 40,8 kg (P50-75) ${ }^{16}$. Radiografia de coluna (Figura 1A) revelou hemivértebras e blocos vertebrais com lordose e escoliose lombar discreta de convexidade para a direita. Ecocardiograma identificou prolapso de valva mitral com fração de ejeção de $80 \%$ e insuficiência mitral leve. Avaliação otorrinolaringológica foi normal. Exames laboratoriais: FSH: 2,88 mUI/mL; LH: $1,84 \mathrm{mUI} / \mathrm{mL}$; relação LH/FSH: 0,64; TSH: 2,1 $\mu \mathrm{UI} / \mathrm{mL}$; T4livre: $1,02 \mathrm{ng} / \mathrm{dL} ;$ Estradiol $\left(\mathrm{E}_{2}\right): 10 \mathrm{pg} / \mathrm{mL}$. Cariótipo em banda G: 50 metáfases 46,XX.

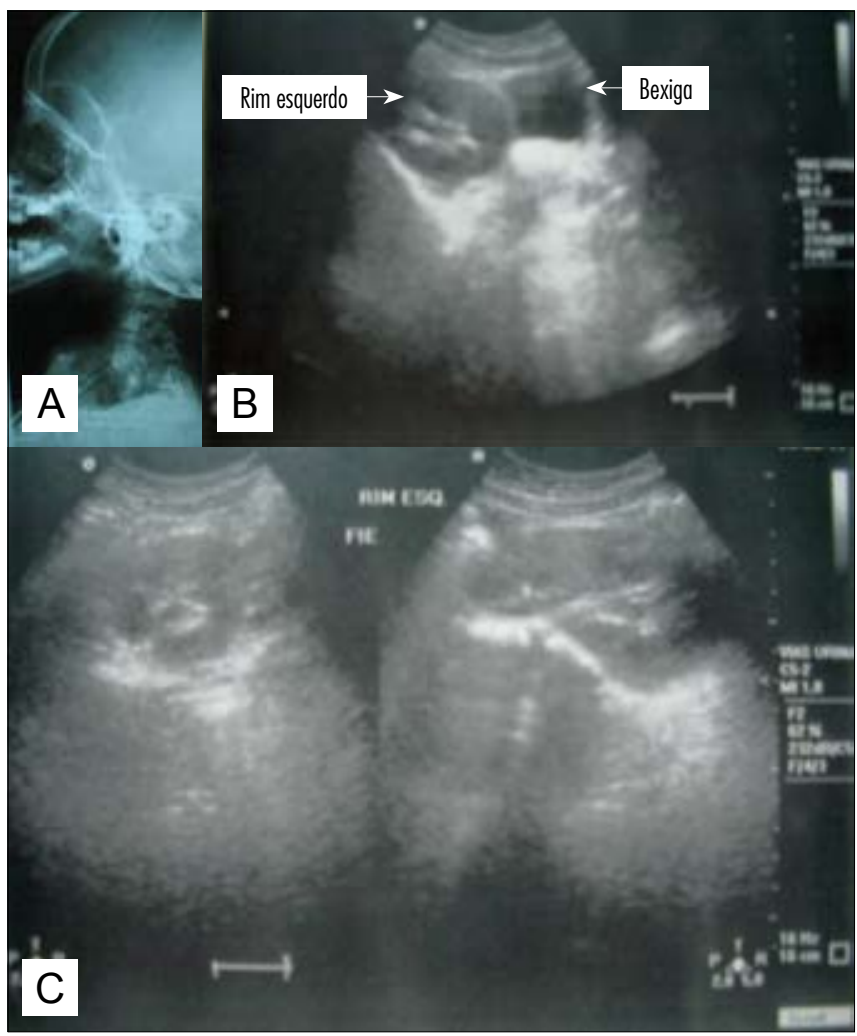

Figura 1. (A) Radiografia de coluna cervical; (B) ultrassonografia pélvica que não evidenciou estruturas müllerianas (útero e trompas); (C) ultrassonografia visualizando rim esquerdo em fossa ilíaca

\section{Segundo caso}

Paciente filha de pais saudáveis, jovens e não consanguíneos iniciou investigação em nosso serviço aos 14 anos por apresentar amenorreia primária. Nasceu de parto vaginal, a termo, com parâmetros antropométricos compatíveis com a idade gestacional. Rim policístico à esquerda, visualizado à USG durante pré-natal, aos 7 meses de gestação, evoluindo para nefrectomia aos 10 anos de idade. Ecocardiograma, aos 8 anos, revelou discreta persistência de canal arterial, valva mitral rudimentar com prolapso do folheto anterior e tendão justo-septal. Ausência de alterações auditivas. Radiografia de coluna demonstrava fusão parcial de C2-C3, hemivértebra em T2, escoliose torácica angular para direita, fusão de arco posterior C6-C7, redução da altura das metades esquerdas entre T1-T2 e T2-T3 (escoliose), ângulo de Cobb na 15ª vértebra torácica. Ao exame físico, apresentava dismorfias faciais discretas e pescoço curto, não caracterizando síndrome genética conhecida. Aos 14 anos, desenvolvimento puberal M-5/G-5, conforme os critérios de Tanner ${ }^{15}$, estatura de $1,54 \mathrm{~m}(\mathrm{P} 25)^{16}$ e peso de $58 \mathrm{~kg}(\mathrm{P} 80)^{16}$, ausência de hipertrofia de clitóris, clinodactilia no quinto quirodáctilo de ambas as mãos. Nessa época, constatou-se: testosterona: $351 \mathrm{pg} / \mathrm{mL}$; FSH: 4,9 mUI/ $\mathrm{mL}$; LH: 5,0 mUI/mL; E: 87 pg/mL; estrona $\left(\mathrm{E}_{1}\right): 109 \mathrm{pg} /$ $\mathrm{mL}$; prolactina: $24 \mathrm{ng} / \mathrm{mL}$; TSH: $5,04 \mu \mathrm{UI} / \mathrm{mL}$; T4 livre: 8,1 ng/dL; anti-TPO: 6,7 UI/mL. USG de pelve: revelou útero rudimentar $(2,0 \times 1,5 \times 0,5 \mathrm{~cm})$ (Figura $2 \mathrm{D})$, presença dos ovários (Figura $2 \mathrm{~A}$ ), rim único à direita (Figura $2 \mathrm{C}$ ); foi complementada pela ressonância magnética (RM) de pelve (Figura 2B), que revelou útero rudimentar. Posterior à uretra, observa-se pequeno canal de $2,5 \mathrm{~cm}$ de comprimento (canal vaginal rudimentar); endométrio não visualizado. TC de crânio sem alterações. Cariótipo: 50 metáfases 46,XX.

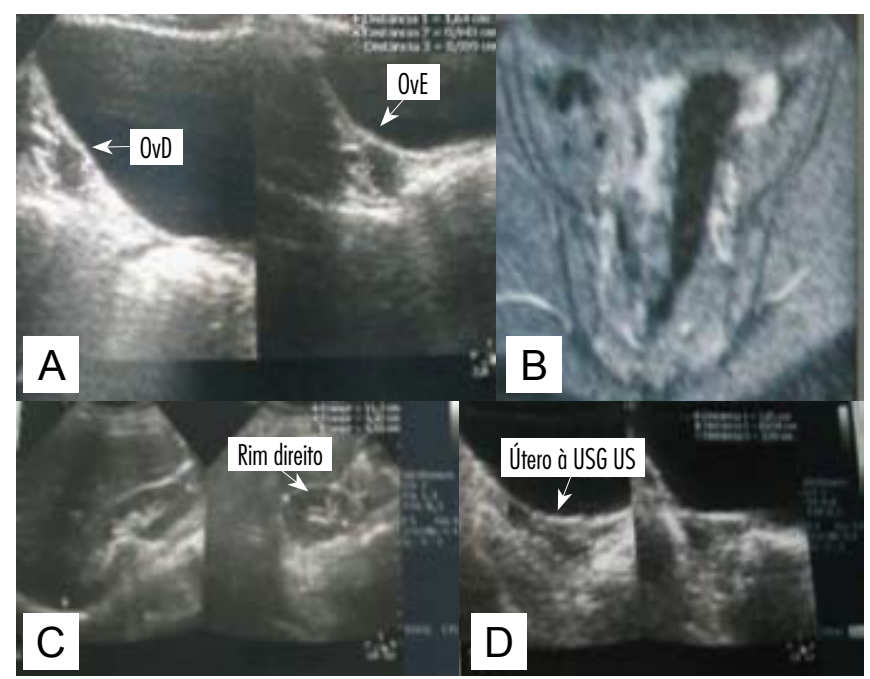

USG: ultrassonografia.

Figura 2. (A) Visualização dos ovários à ultrassonografia pélvica; (B) visualização do útero rudimentar à ressonância magnética pélvica $(C)$ evidenciado ausência do rim esquerdo e visualização do rim direito à ultrassonografia abdominal; (D) visualização do útero rudimentar $(2,0 \times 1,5 \times 0,5 \mathrm{~cm})$ pela ultrassonografia pélvica 


\section{Discussão}

É de suma importância que MURCS faça parte da avaliação diagnóstica diferencial em pacientes do sexo feminino com malformações renais, genitais e/ou de coluna, que apresentem amenorreia primária, tendo em vista que quanto mais precoce o diagnóstico, mais adequada será a conduta ${ }^{2,6,9}$.

No primeiro caso relatado, foi possível estabelecer o diagnóstico precoce da associação de MURCS, pois a paciente foi encaminhada ao ambulatório de genética aos 6 anos de idade, apresentando deformidades na coluna cervical, tipo Klippel-Feil e história de malformação renal, sendo solicitada e realizada a USG pélvica, que não evidenciou estruturas müllerianas (Figura 1B). A confirmação diagnóstica foi realizada pelo cariótipo normal e pelo fato de também não terem sido encontradas outras comorbidades associadas.

A paciente adolescente já poderia ter tido esse diagnóstico confirmado anteriormente, pois apresentou malformação renal, sendo submetida à nefrectomia aos 10 anos, mas a investigação não prosseguiu. Foi encaminhada à endocrinologia somente aos 14 anos, por apresentar amenorreia primária, apesar do desenvolvimento normal dos caracteres sexuais secundários. Foi, então, solicitada investigação do esqueleto, que mostrou fusão de vértebras e RM pélvica (Figura 2B), que detectou útero rudimentar com agenesia de vagina. $\mathrm{O}$ cariótipo também foi normal.

Anomalias ocasionais incluem baixa estatura, defeitos nas costelas, defeitos em membros superiores, assim como anomalia escapular de Sprengel. Nossas pacientes, porém, não apresentavam nenhum desses defeitos. Achados incomuns incluem surdez, cisto cerebelar, defeitos de ouvido externo, assimetria facial, lábio leporino, fenda palatina, micrognatia, defeito gastrintestinal e defeitos de lateralidade ${ }^{17}$. Ambas foram submetidas às avaliações auditivas e ecocardiográficas e, apesar de não apresentarem déficit auditivo, havia em comum prolapso de válvula mitral. Não há relato na literatura sobre a frequência de determinados defeitos cardíacos mais comuns ou específicos à associação de MURCS ${ }^{18}$.

Para o ginecologista e o endocrinologista, a amenorreia primária será o aspecto preponderante, e a integridade da genitália interna e externa deve fazer parte da investigação inicial. Confirmada a ausência ou hipoplasia de útero e vagina, deve ser realizada a dosagem hormonal, a fim de se estabelecer o diagnóstico diferencial de MURCS ${ }^{2,6,9}$ com as disgenesias gonadais, cujos resultados são anormais quando comparados àqueles encontrados na portadora da associação de MURCS. Outro aspecto a ser levado em conta é a história familiar, na qual a presença de outros casos semelhantes ${ }^{9}$, e a existência de malformações renais e de esqueleto em parentes de primeiro e segundo graus deve ser investigada, para confirmar ou excluir as formas herdadas ${ }^{8,19} \mathrm{e}$, com isso, possibilitar a investigação molecular, a fim de estabelecer qual é a mutação gênica envolvida e propiciar o aconselhamento genético quando pertinente.

A história gestacional também deve ser considerada, pois há correlação entre MURCS e diabetes gestacional ${ }^{6}$, assim como por uso de talidomida na gravidez ${ }^{6,9}$; ambos são fatores de risco para várias outras doenças. Não foi evidenciada a associação desses fatores em nossas pacientes.

Uma vez identificada a hipoplasia ou agenesia de estruturas müllerianas, a USG abdominal total deve ser realizada a fim de identificar anomalias renais ${ }^{10,18}$. No entanto, a detecção de estrutura quadrangular retrovesical pode, erroneamente, ser identificada como útero hipoplásico ou juvenil, o que, na verdade, corresponde a vestígio laminar, localizado embaixo do peritônio e transversalmente ao lado posterior da bexiga, no qual se fixam os ligamentos uterossacrais ${ }^{6}$. A USG morfológica pode ser útil no diagnóstico pré-natal, se deformidades esqueléticas, renais ou cardíacas forem detectadas ${ }^{19}$. Nossas pacientes apresentaram alterações renais à USG pré-natal, sem evidência de outras malformações. Na fase pós-natal, confirmou-se, por esse exame, rim único e pélvico em uma paciente, e rim cístico com refluxo urétero-renal, que culminou em nefrectomia, na adolescente. A avaliação da morfologia da genitália interna foi consequência da deformidade de Klippel-Feil e da presença de rim único e ectópico na criança, hoje ciente de não apresentar menarca; a $\mathrm{RM}$ foi fundamental para definir o aspecto morfológico interno da paciente adolescente ${ }^{6}$, o que não foi possível à USG, porém a RM, por ser exame não invasivo, prepondera sobre a celioscopia, procedimento invasivo e, por vezes, necessário, quando, por exemplo, houver suspeita de disgenesia gonadal XY, na qual a retirada das gônadas se impõe ${ }^{6}$.

O diagnóstico diferencial depende da idade e da presença do achado clínico. $\mathrm{Na}$ infância, as síndromes de Turner e Noonan devem ser descartadas, pois ambas levam a alterações cardíacas, esqueléticas e renais, mas a primeira condição apresenta cariótipo anormal, enquanto o hipogonadismo é encontrado em ambas as patologias. $\mathrm{Na}$ adolescência, o espectro de patologias é mais amplo e requer exame físico cuidadoso e a constatação de amenorreia primária, a fim de excluir disgenesias gonadais puras $\mathrm{XX}$ ou XY, assim como insensibilidade a androgênios; nesses dois últimos casos, o cariótipo estabelece o diagnóstico e a conduta terapêutica é cirúrgica ${ }^{2,9}$.

Em geral, o tratamento depende da gravidade das malformações ${ }^{8}$. Do ponto de vista ginecológico, o tratamento é conservador e adiado até que a paciente esteja 
pronta para iniciar a atividade sexual. $\mathrm{O}$ método usado deve ser avaliado individualmente, levando sempre em consideração a escolha da paciente, podendo incluir intervenção cirúrgica ou não.

Quando a paciente descobre a presença de malformação congênita envolvendo seus órgãos reprodutores, é comum o desenvolvimento de ansiedade e angústia sobre sua feminilidade, aparência física ${ }^{6,9}$ e capacidade reprodutora, sendo fundamental apoio psicoterápico para a paciente, assim como para seus familiares.

A associação de MURCS pode ser atribuída a alterações no blastema, que dão origem aos somitos cervicotorácicos e o ducto paramesonéfrico, cujas relações espaciais já estão determinadas na embriogênese até a quarta semana ${ }^{1}$. A possibilidade de heterogeneidade genética na etiologia da associação de MURCS levou os pesquisadores a buscarem o gene ou genes nela envolvidos, assim como a região cromossômica onde estes se localizam. Alguns estudos têm se mostrado promissores quanto a isso, por exemplo, o gene WNT4, localizado no braço curto do cromossomo 1, expresso precocemente no mesonéfron em desenvolvimento, que está envolvido no desenvolvimento das trompas de Falópio, útero, ovários e glândulas mamárias. Mutações do gene WNT4 têm sido associadas à malformação dos ductos de Müller e virilização (hiperandrogenismo) em mulheres com cariótipo $46, \mathrm{XX}^{20}$, ao contrário de mutações localizadas no braço longo do cromossomo 22, cujos resultados são controversos quanto à sua associação com MURCS ${ }^{1,20}$. Apesar da pesquisa molecular do gene ou genes envolvidos não ter sido realizada no presente estudo, a documentação e o relato de casos contribuem para o conhecimento de sua frequência em nosso meio.

Portanto, quanto maior o número de casos descritos, mais possibilidades de se identificar a etiologia da associação de MURCS, inclusive com melhor definição do papel da mutação no desenvolvimento dos órgãos afetados e, a partir daí, poderá ser possível estabelecer o risco de recorrência e, consequentemente, propiciar o aconselhamento genético para as pacientes e suas famílias.

Concluímos que o diagnóstico precoce da associação de MURCS é possível, desde que se investiguem outras malformações na vigência de fusão de vértebras ou malformação renal, sabendo que a amenorreia primária pode estar associada e, consequentemente, proporcionar apoio e orientação para pacientes e seus familiares, além de traçar a melhor forma de condução do caso e propiciar o aconselhamento genético.

\section{Referências}

1. Morcel K, Watrin T, Pasquier L, Rochard L, Le Caignec C, Dubourg C, et al. Utero-vaginal aplasia (Mayer-Rokitansky-Küster-Hauser syndrome) associated with deletions in known DiGeorge or DiGeorge-like loci. Orphanet J Rare Dis. 2011 ;6:9.

2. Saad Ganem A, López-Ortiz CG, Lejtik Alva CA, Quintero Bernal P, Zafra de La Rosa G, Gutiérrez Palomares V. Asociación MURCS: reporte de un caso. Ginecol Obstet Mex. 2008;76(6):349-52.

3. Hofstetter G, Concin N, Marth C, Rinne T, Erdel M, Janecke A. Genetic analyses in a variant of Mayer-Rokitansky-KusterHauser syndrome (MURCS association). Wien Klin Wochenschr. 2008;120(13-14):435-9.

4. Balusamy SL, Kumar D, Subrahmanian SR. MURCS and VACTERL association in a 27 year old female. J Obstet Gynaecol. 2009;29(8):762-3.

5. Al Kaissi A, Ben Chehida F, Ben Ghachem M, Grill F, Klaushofer K. Occipitoatlantoaxial junction malformation and early onset senile ankylosing vertebral hyperostosis in a girl with MURCS association. Am J Med Genet A. 2009; 149A(3):470-4.

6. Morcel K, Camborieux L, Guerrier D; Programme de Recherchessur les Aplasies Müllérienes (PRAM). Mayer-Rokitansky-Küster-Hauser (MRKH) syndrome. Orphanet J Rare Dis. 2007;2:13.

7. Greene RA, Bloch M, Huff DS, lozzo RV. MURCS association with additional congenital anomalies. Hum Pathol. 1986; $17(1): 88-91$
8. Oppelt P, Renner SP, Kellermann A, Brucker S, Hauser GA, Ludwig $\mathrm{KS}$, et al. Clinical aspect of Mayer-Rokitansky-Küster-Hauser syndrome: recommendations for clinical diagnosis and staging. Hum Reprod. 2006;21(3):792-7.

9. Guerrier D, Mouchel T, Pasquier L, Pellerin I. The Mayer-RokitanskyKüster-Hauser syndrome (congenital absence of uterus and vagina) - phenotypic manifestations and genetic approaches. J Negat Results BioMed. 2006;5:1.

10. Pittock ST, Babocic-Vuksanovic D, Lteif A. Mayer-Rokitansky-KüsterHauser anomaly and its associated malformations. Am J Med Genet A. 2005;135(3):314-6.

11. Linke F, Kraemer W, Ansorge M, Brzezinska R, Berger S. Right esophageal lung in a preterm child with VACTERL association and Mayer-RokitanskyKüster-Hauser syndrome. Pediatr Surg Int. 2005;21 (4):285-8.

12. Suri $M$, Brueton LA, Venkatraman N, Cox PM. MURCS association with encephalocele: report of a second case. Clin Dysmorphol. 2000;9(1):31-3.

13. Lin HJ, Cornford ME, Hu B, Rutgers JK, Beall M, Lachman RS. Occipital encephalocele and MURCS association: case report and review of central nervous system anomalies in MURCS patients. Am J Med Genet. 1996;61(1):59-62.

14. Lo ludice G, Federico P, Pasquali D, Petrenga E, Schillirò F, D'Alessandro B. The MURCS association. Clinical, radiological, endocrinological and familial data in a 40-year old patient. Minerva Endocrinol. 1986;1 1(3):205-9. 
15. Kochi C. Critérios de avaliação da puberdade. In: Monte $O$, Longui CA, Calliari LE, Kochi C, organizadores. Endocrinologia para o pediatra. 3a ed. São Paulo: Atheneu; 2006. p. $151-6$.

16. Marques RM, Marcondes E, Berquó E, Prandi R, Yunes J. Crescimento e desenvolvimento pubertário em de crianças e adolescentes brasileiros II: altura e peso. São Paulo: Editora Brasileira de Ciências; 1982.

17. Jones KL. Smith's recognizable patterns of human malformation. 5th ed. Philadelphia: WB Saunders; 1997. p. 666-7.
18. Carranza-Lira S, Forbin K, Martinez-Chéquer JC. Rokitansky syndrome and MURCS association-clinical features and basis for diagnosis. Int J Fertil Womens Med. 1999;44(5):250-5.

19. Pelizzo G, Lembo MA, Civitelli S, La Riccia A, Franchella A. Prenatal management of inherited urogenital malformation: case report. Clin Exp Obstet Gynecol. $2001 ; 28(1): 31$-2.

20. Philibert $P$, Biason-Lauber $A$, Rouzier R, Pienkowski C, Paris F, Konrad $D$, et al. Identification and functional analysis of a new wnt4 gene mutation among 28 adolescent girl with primary amenorrhea and müllerian duct abnormalites: a French Collaborative Study. J Clin Endocrinol Metab. 2008;93(3):895-900. 